\title{
Do FLAIR Vascular Hyperintensities beyond the DWI Lesion Represent the Ischemic Penumbra?
}

\author{
L. Legrand, M. Tisserand, G. Turc, O. Naggara, M. Edjlali, C. Mellerio, J.-L. Mas, J.-F. Méder, J.-C. Baron, and C. Oppenheim
}

\begin{abstract}
BACKGROUND AND PURPOSE: In acute stroke with proximal artery occlusion, FLAIR vascular hyperintensities observed beyond the boundaries of the cortical lesion on DWI (newly defined "FLAIR vascular hyperintensity-DWI mismatch") may be a marker of tissue at risk of infarction. Our aim was to compare the occurrence of FLAIR vascular hyperintensity-DWI mismatch relative to that of perfusionweighted imaging-DWI mismatch in patients with proximal MCA occlusion before IV thrombolysis.
\end{abstract}

MATERIALS AND METHODS: In 141 consecutive patients with proximal MCA occlusion, 2 independent observers analyzed FLAIR images for the presence of FLAIR vascular hyperintensity-DWI mismatch before IV thrombolysis. PWI-DWI mismatch was defined as Volume $_{\text {hypoperfusion }}>1.8 \times$ Volume $_{\mathrm{DW}}$, with Volume hypoperfusion $>6$ seconds on time to maximum value of the residue function maps in the 94 patients with available PWI. The presence of FLAIR vascular hyperintensity-DWI mismatch, PWI-DWI mismatch, and infarct growth on 24-hour follow-up DWI was compared.

RESULTS: A FLAIR vascular hyperintensity-DWI mismatch was present in 102/141 (72\%) patients, with an excellent interobserver reliability $(\kappa=0.91)$, and a PWI-DWI mismatch, in 61 of the $94(65 \%)$ patients with available PWI. FLAIR vascular hyperintensity-DWI mismatch predicted PWI-DWI mismatch with a sensitivity of $92 \%(95 \% \mathrm{Cl}, 85 \%-99 \%)$ and a specificity of $64 \%(95 \% \mathrm{Cl}, 47 \%-80 \%)$. Patients with FLAIR vascular hyperintensity-DWI mismatch had smaller initial DWI lesion and larger infarct growth $(P<.001)$ than patients without FLAIR vascular hyperintensity-DWI mismatch, even though their final infarcts remained smaller $(P<.001)$.

CONCLUSIONS: Albeit being moderately specific, probably due to inclusion of oligemic tissue, the FLAIR vascular hyperintensity-DWI mismatch identifies large PWI-DWI mismatch with high sensitivity.

ABBREVIATIONS: $\mathrm{DWI}_{1}=$ pretreatment lesions on $\mathrm{DWl}$; $\mathrm{DWI}_{2}=$ follow-up lesions on $\mathrm{DWI} ; \mathrm{FVH}=$ FLAIR vascular hyperintensity; $\mathrm{Tmax}=$ time to maximum value of the residue function

A dvances in MR imaging have recently allowed better characterization of tissue and vessel status in acute stroke. FLAIR sequences are part of acute stroke MR imaging protocols in institutions using MR imaging as the first-line diagnostic tool. ${ }^{1}$ In normal conditions, intracranial arteries are dark on FLAIR due to

Received May 27, 2014; accepted after revision June 29.

From the Departments of Radiology (L.L., M.T., O.N., M.E., C.M., J.-F.M., C.O.) and Neurology (G.T., J.-L.M, J.-C.B.), Université Paris Descartes Sorbonne Paris Cité, Centre de Psychiatrie et Neurosciences, Institut National de la Santé et de la Recherche Médicale S894, Centre Hospitalier Sainte-Anne, Paris, France.

Laurence Legrand is supported by the Société Française de Radiologie; Marie Tisserand is supported by the Fondation de la Recherche Médicale.

Paper previously presented at: Annual Meeting of the American Society of Neuroradiology and the Foundation of the ASNR Symposium, May 17-22, 2014; Montreal, Quebec, Canada.

Please address correspondence to Catherine Oppenheim, MD, PhD, Service d'Imagerie, Centre Hospitalier Sainte-Anne, 1 Rue Cabanis, 75674 Paris Cedex 14, France; e-mail: c.oppenheim@ch-sainte-anne.fr

http://dx.doi.org/10.3174/ajnr.A4088 the flow-void phenomenon caused by a loss of signal intensity produced by the movement of blood. After an intracranial arterial occlusion, FLAIR vascular hyperintensities (FVHs) are seen in $45 \%-100 \%$ of patients with stroke. ${ }^{1-14}$ Although their pathophysiologic and clinical significance is not fully understood, FVHs might help in the management of patients with acute stroke. Beyond their diagnostic value regarding the detection of arterial occlusion, ${ }^{11,14,15}$ FVHs may provide prognostic information, despite discrepancies among studies. ${ }^{1}$ Some authors reported that FVHs have a good prognostic value ${ }^{3-6}$; some, that they have a poor prognostic value ${ }^{7-10}$; and others, that they have no prognostic value at all. ${ }^{11}$ These discrepancies are likely due to differences among populations, end points, and FVH classifications.

Meanwhile, most groups agree that FVHs are related, to some extent, to hemodynamic impairment and represent slow retrograde flow in leptomeningeal collaterals. ${ }^{2}$ These collaterals maintain some perfusion distal to the occlusion while awaiting revascularization. ${ }^{16}$ FVHs precede DWI abnormalities ${ }^{17}$ and can be 
seen beyond the boundaries of the DWI lesion, and the final infarct volume falls between the initial DWI lesion and the area facing the FVHs. ${ }^{14}$ Furthermore, FVHs beyond the clot are associated with perfusion-weighted imaging-DWI mismatch ${ }^{3,8,13}$ and better outcome after IV-tPA. ${ }^{3}$ Taken together, these data suggest that prominent/extended FVHs indicate large areas of salvageable tissue and greater potential benefit from recanalization. In patients with stroke with acute proximal MCA occlusion, we tested the hypothesis that FVHs beyond the DWI cortical lesion boundaries (termed "FVH-DWI mismatch") could be an easy and reproducible means to identify patients with a large penumbral area (ie, with a large PWI-DWI mismatch) and larger infarct growth.

\section{MATERIALS AND METHODS \\ Patients}

This retrospective analysis was based on a prospectively collected monocenter registry of consecutive patients exclusively treated by IV-tPA $(0.9 \mathrm{mg} / \mathrm{kg})$ for ischemic stroke between 2004 and 2013. MR imaging is systematically implemented in our center as a firstline pretherapeutic work-up in candidates for thrombolysis. They were treated within 3 hours from stroke onset until November 2008 and within 4.5 hours thereafter. Patients included in the present study were those who had the following: 1) an anterior circulation stroke due to proximal MCA occlusion (M1 segment); 2) pretreatment MR imaging; and 3) at least a PWI sequence before treatment or a 24-hour follow-up MR imaging, given that we searched for associations between FVH-DWI mismatch and PWI-DWI mismatch or between FVH-DWI mismatch and infarct growth. Internal carotid artery occlusion was not an exclusion criterion as long as the ipsilateral anterior cerebral artery remained visible on MRA. Patients with severe MR imaging artifacts or MR images not available in DICOM format were excluded.

Age, sex, hypertension, diabetes mellitus, hyperlipidemia, smoking, arterial blood pressure, serum glucose level at admission, and National Institutes of Health Stroke Scale score before and 24 hours after treatment were prospectively collected. Outcome at 3 months was assessed by using the mRS. Stroke etiology was assessed by using Trial of Org 10172 in Acute Stroke Treatment classification. The study was approved by the local ethics committee.

\section{MR Imaging Protocol}

Pretreatment and follow-up MR imaging was performed on 1.5T scanners (Signa Horizon EchoSpeed until November 2008 and Signa EchoSpeed thereafter; GE Healthcare, Milwaukee, Wisconsin) with a $33-\mathrm{mT} / \mathrm{m}$ gradient strength and an 8-channel head coil. The standard MR imaging protocol included FLAIR, DWI, $\mathrm{T} 2{ }^{*}$-weighted gradient-echo imaging, intracranial 3D time-offlight MRA, and PWI whenever feasible with no delay. Total acquisition time was $\leq 10$ minutes The acquisition parameters of the axial 2D FLAIR sequence were as follows: TR/TE/TI, 82779802/155.5-159.4/2093-2300 ms; $24 \times 24 \mathrm{~cm}^{2}$ FOV; $256 \times 192$ matrix; 1 excitation; 24 sections; 6 -mm contiguous section thickness; maximum duration, 2 minutes 18 seconds. DWI consisted of a single-shot echo-planar spin-echo sequence ( 3 directions, $b=$
$1000 \mathrm{~s} / \mathrm{mm}^{2}$ ). PWI was performed by using a $2^{\star}$-weighted echoplanar sequence (TR/TE, 2000/60 ms; $24 \times 24 \mathrm{~cm}^{2} \mathrm{FOV} ; 64 \times 96$ matrix; 1 excitation; and repetition 50 times after a bolus [5-7 $\mathrm{mL} / \mathrm{s}$ ] of $20 \mathrm{~mL}$ of gadoteric acid). A follow-up MR imaging was scheduled $\sim 24$ hours after tPA and included the same set of sequences as that in the admission MR imaging except for PWI. All MR images were processed on a commercial workstation (Advantage Windows; GE Healthcare) by using an application for functional mapping calculation (READY View; GE Healthcare).

\section{Image Analysis}

FVHs were defined as focal, tubular, or serpentine hyperintensities in the subarachnoid space relative to $\mathrm{CSF}^{1}$ and corresponding to the typical arterial course. Axial FLAIR and DWI were reviewed together to assess a FVH-DWI mismatch. FVH-DWI mismatch was considered present when FVHs extended beyond the boundaries of the cortical DWI lesion (ie, when $\geq 1 \mathrm{FVH}$ was facing the isointense cortex on DWI). FVH-DWI mismatch was considered absent when there was no FVH or when all FVHs were facing the hyperintense cortex on DWI. Two radiologists (L.L. and C.O., with 3 and 17 years' experience in stroke imaging, respectively) independently reviewed the image datasets on a dedicated workstation for FVH-DWI mismatch evaluation. They were aware that all included patients had a proximal MCA occlusion; they had access to pretreatment DWI, MR angiography, and T2* images; but they were blinded to PWI, clinical data, and follow-up. Discordance between observers was resolved by consensus. One neuroradiologist (L.L.) reviewed the $\mathrm{T} 2 *$ images to ensure that FVHs did not correspond to blood clots and to evaluated FLAIR image quality (good-to-excellent or diagnostic quality despite artifacts).

Pretreatment $\left(\mathrm{DWI}_{1}\right)$ and follow-up DWI $\left(\mathrm{DWI}_{2}\right)$ lesions were segmented to compute initial and final DWI lesion volumes as detailed elsewhere, ${ }^{18}$ and $\mathrm{DWI}_{1}$ lesion extent was evaluated semiquantitatively by using the 10-point DWI-ASPECTS. Relative infarct growth was defined as Volume $_{\text {DWI2 }} /$ Volume $_{\text {DWI1 }} \times 100$. Critically hypoperfused tissue, defined as time to maximum value of the residue function (Tmax) $>6$ seconds, was segmented from PWI maps (BrainStat AIF, READY View software; GE Healthcare). The PWIDWI mismatch volume was defined as the Tmax $>6$-second volume without $\mathrm{DWI}_{1}$ changes. On the basis of Diffusion and Perfusion Imaging Evaluation for Understanding Stroke Evolution (DEFUSE 2) criteria, a PWI-DWI mismatch was considered present when Volume $_{\text {hypoperfusion }}$ exceeded $1.8 \times$ Volume $_{\mathrm{DWII}} \cdot{ }^{19}$ This stringent ratio was preferred to the classic 1.2 ratio to select patients with clinically meaningful PWI-DWI mismatch only. Pretreatment occlusion and recanalization (TICI score) were assessed on MRA.

For the per-region analysis, FVHs were rated by 2 readers according to their distribution based on the ASPECTS regions. Briefly, the 7 cortical divisions of ASPECTS (insula, M1-M6) were considered positive when they coincided with an FVH. Of note, as opposed to the original ASPECTS and its previous adaptation to FVHs in which only 2 axial sections were selected, ${ }^{5,10,20}$ we reviewed all FLAIR images here to ensure continuity of bright dots considered FVHs. Coregistered $\mathrm{DWI}_{1}, \mathrm{DWI}_{2}$, and PWI (Tmax $>6$-second maps) were analyzed visually by 1 reader for DWI hyperintensity, infarct progression, and hypoperfusion in each of the 7 ASPECTS regions. Each 


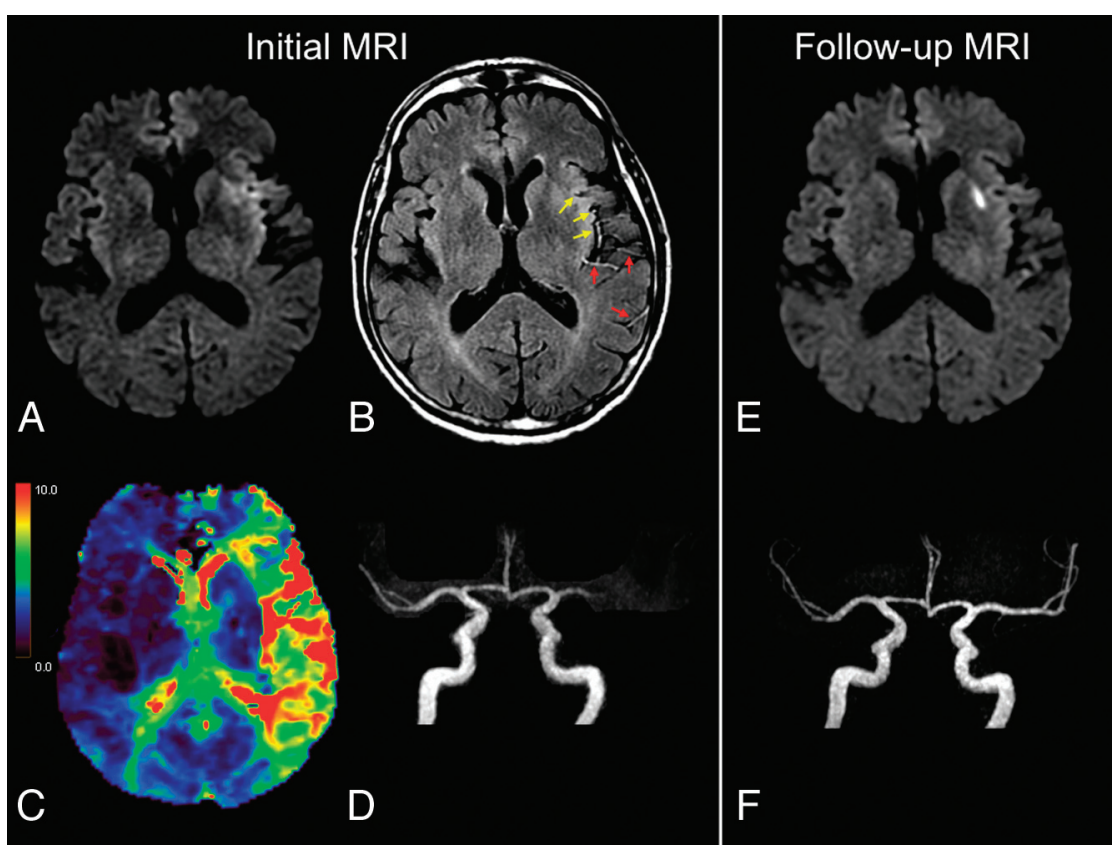

FIG 1. Illustrative case of FVH-DWI and PWI-DWI mismatch. MR images $(A-D)$ of a 67-year-old man obtained 86 minutes after sudden onset of aphasia. Small hyperintense lesions are visible in the left MCA territory on admission DWI (A), with FVHs on FLAIR (B), some facing the DWI lesion (yellow arrows) and others located beyond the boundaries of DWI signal changes (red arrows), indicating a FVH-DWI mismatch. A large PWI-DWI mismatch on the Tmax map (C) with areas of Tmax of $>6$ seconds (yellow-to-red) topographically congruent with the FVHs. Proximal occlusion of left MCA on MRA ( $D$, frontal view). On 24-hour follow-up MR images ( $E$ and $F$ ), there was no extension of the initial DWI lesion (E) and complete recanalization (F). IV-tPA was initiated 120 minutes after stroke onset.

ASPECTS region was subsequently classed as positive or negative for FVH-DWI and PWI-DWI mismatch.

\section{Statistical Analysis}

We compared continuous variables using the Student $t$ test or Mann-Whitney $U$ test, as appropriate. Categoric variables were compared by using the $\chi^{2}$ or Fisher exact test as appropriate. The $\kappa$ coefficient was used to assess interobserver agreement for FVHDWI mismatch. We compared pre- and post-treatment characteristics in univariate analyses between patients with or without FVH-DWI mismatch and PWI-DWI mismatch. We finally searched for a model that would predict PWI-DWI mismatch on the basis of clinical and easily derivable imaging parameters avail-

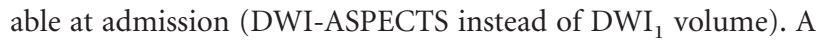
multivariate binary logistic regression analysis was performed with PWI-DWI mismatch (present/absent) as the dependent variable. Variables were selected for entry into the model on the basis of results of the univariate analysis $(P<.20)$ and were further excluded from the model with $P<.10$. The OR and 95\% CI were obtained. A 2-tailed $P$-value $<.05$ was considered significant (SPSS for Windows, Version 19.0; IBM, Armonk, New York). Sensitivity, specificity, and positive and negative predictive values of the FVH-DWI mismatch for the detection of PWI-DWI mismatch were computed.

\section{RESULTS}

\section{General Population}

During the study period, 351 patients were treated with IV-tPA only. Two hundred ten (60\%) patients were excluded for the fol- lowing main reasons: non-MCA territory stroke $(n=38)$, CT scan at admission $(n=32)$, initial MR imaging not available in DICOM format $(n=11)$ or noninterpretable ( $n=11$, major artifacts), absence of M1 occlusion ( $n=$ 110), M1 occlusion combined with ipsilateral internal carotid and anterior cerebral artery occlusion $(n=2)$, and no PWI sequence or follow-up MR imaging $(n=6)$. One hundred forty-one patients (74 men) met the inclusion criteria, with a median (interquartile range) age and admission NIHSS score of 70 years (range, 57-79 years) and 17 (range, 1221), respectively. They did not differ from the excluded patients on the basis of sex $(P=.34)$ or age $(P=.81)$ but had a higher NIHSS score of 17 (range, 12$21)$ versus 11 (range, $7-18)(P<.001)$. PWI was available in $94(67 \%)$ patients. Median time-to-initial MR imaging and time to treatment were 117 minutes (range, 89-157 minutes) and $155 \mathrm{~min}$ utes (range, 123-194 minutes). Follow-up MR imaging (median [interquartile range] delay from onset $=25$ hours [21-29 hours]) was available in 138 patients. Of these, complete recanalization (TICI 3) occurred in 41 patients (30\%). The 3-month mRS score was available in 133 patients, with a median score of 3 (range, 1-4).

\section{FVH-DWI Mismatch (Per-Patient Analysis)}

The interobserver agreement for FVH-DWI mismatch (Figs 1 and 2) was $\kappa=0.91$ (95\% CI, 0.84-0.99). After consensus, 102 (72\%) patients had a FVH-DWI mismatch. As shown in the Table, patients with FVH-DWI mismatch had a smaller DWI $_{1}$ volume and a higher PWI-DWI mismatch volume than patients without FVH-DWI mismatch. Patients with FVH-DWI mismatch had greater infarct progression at follow-up MR imaging, even though their follow-up infarct volume remained smaller and 3-month outcome was better than that of patients without FVH-DWI mismatch. All the above-mentioned associations remained significant when the analysis was restricted to patients with good-toexcellent FLAIR image-findings quality ( $n=105$ in the whole population, $n=74$ in patients with PWI). The presence of FVHDWI mismatch did not differ between patients treated before and after November $2008(37 / 52=71 \%$ versus $65 / 89=73 \%, P=$ $.81)$.

\section{Per-Region Analysis}

FVHs were visible in the insular region in all cases and were facing the M2, M5, M3, M6, M1, and M4 ASPECTS regions in 95\%, $75 \%, 71 \%, 37 \%, 24 \%$, and $15 \%$ of patients, respectively (average of 2 readers). Of the 987 ASPECTS regions (141 patients $\times 7$ regions), 60\% were facing an FVH. In line with the per-patient 


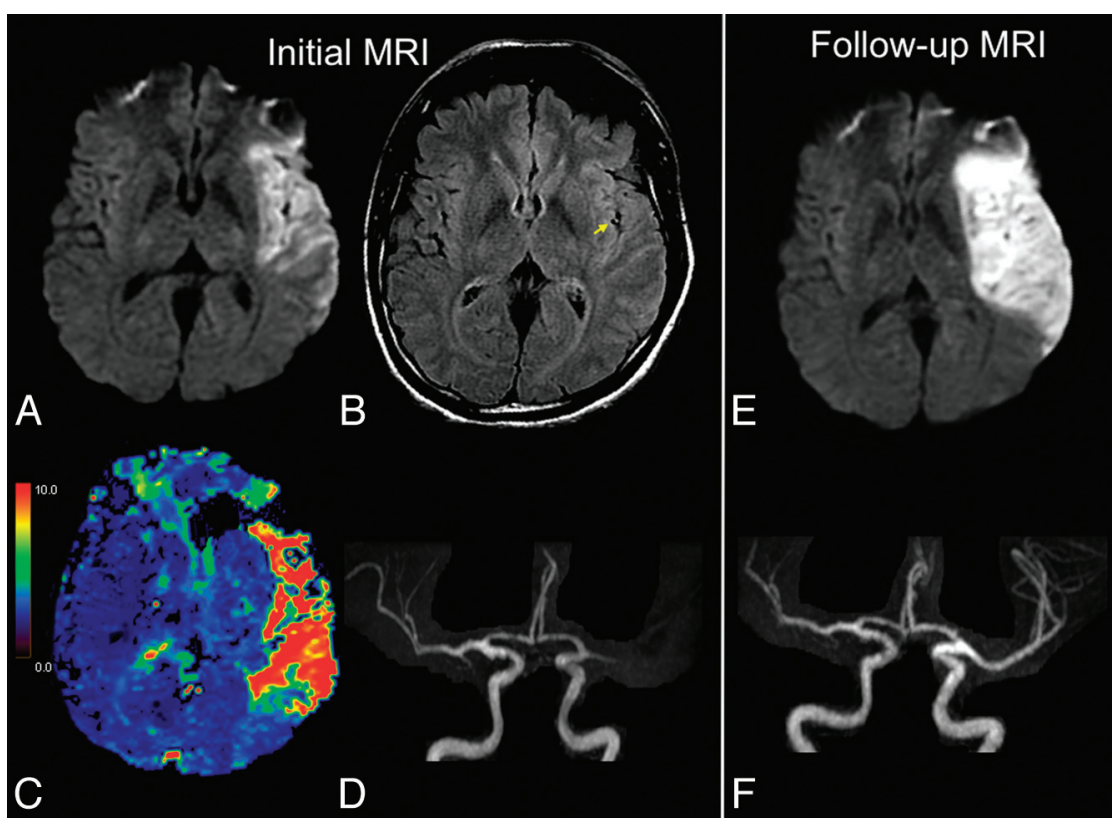

FIG 2. Illustrative case of no FVH-DWI or PWI-DWI mismatch. MR imaging $(A-D)$ of a 47-year-old man obtained 81 minutes after sudden onset of right hemiparesis. Large hyperintense lesions in the left MCA territory on admission DWI $(A)$ with FVH on FLAIR ( $B$, arrow) only overlying the hyperintense parenchyma on DWI, indicating the absence of FVH-DWI mismatch. $C$, No significant PWI-DWI mismatch on the Tmax map is seen. Note proximal occlusion of the left MCA on MRA ( $D$, frontal view). On 24-hour follow-up MR images ( $E$ and $F$ ), extension of the initial DWI lesion (E) is seen despite recanalization (F). IV-tPA was initiated 105 minutes after stroke onset. match $($ specificity $=64 \% ; 95 \% \mathrm{CI}$, $47 \%-80 \%)$. The positive and negative predictive values were $82 \%(95 \% \mathrm{CI}$, $73 \%-91 \%)$ and $81 \%(95 \% \mathrm{CI}, 65 \%-$ $96 \%)$, respectively. A post hoc analysis by using a less conservative threshold for the PWI-DWI mismatch profile (4-second Tmax threshold instead of 6-second) resulted in a similar sensitivity but a higher specificity $(90 \%$ and $76 \%$, respectively).

\section{DISCUSSION}

The present study revealed 3 salient points: 1) FVHs were consistently visualized in the insular region in patients with proximal MCA occlusion within 4.5 hours after symptom onset; 2) FVHDWI mismatch was associated with a smaller initial infarct, the presence of PWI-DWI mismatch, and greater infarct growth after thrombolysis even though their final infarcts remained smaller; and 3) FVH-DWI mismatch predicted a large PWI-DWI mismatch with excellent sensitivity but moderate specificity.

analysis (see next paragraph), FVH-DWI mismatch was significantly associated with PWI-DWI mismatch: Eighty-two percent of regions positive for FVH-DWI mismatch were also positive for PWI-DWI mismatch versus only $17 \%$ of regions negative for FVH-DWI mismatch $(P<.001)$. Infarcts progressed more often in brain tissue facing FVHs: Thirty-one percent of the areas with FVHs showed infarct progression versus $21 \%$ of the areas without it $(P<.001)$.

\section{Identification of PWI-DWI Mismatch}

A PWI-DWI mismatch was present in 61 of the 94 (65\%) patients with available PWI. In univariate analysis, patients with PWIDWI mismatch had lower initial NIHSS scores (15 [range, 11-20] versus 19 [range, 16-23], $P=.003$ ), smaller $\mathrm{DWI}_{1}$ lesion extent assessed by using volumes (17 mL [range, 7-35 mL] versus 108 $\mathrm{mL}$ [range, $63-149 \mathrm{~mL}$ ], $P<.001$ ) or DWI-ASPECTS $(7$ [range, $6-8$ ] versus 4 [range, $3-6$ ],$P<.001$ ), shorter onset-to-initial MR imaging time (106 minutes [range, $84-137$ minutes] versus 132 minutes [range, 97-178 minutes], $P=.01$ ), and lower serum glucose levels at admission $(6.7 \pm 1.2$ versus $7.3 \pm 1.8 \mathrm{mmol} / \mathrm{L}$, $P=.04)$. In multivariate analysis based on easily derivable admission variables, PWI-DWI mismatch was independently associated with FVH-DWI mismatch (OR, 7.63; 95\% CI, 1.74-33.43; $P=$ .007) after adjustment for onset-to-admission MR imaging time (OR, 0.98; 95\% CI, 0.97-1.00; $P=.02$ ) and DWI-ASPECTS (OR, $1.76 ; 95 \%$ CI, 1.22-2.53; $P=.003)$. Initial NIHSS score and serum glucose level were sequentially removed from the model.

Of the 94 patients with available PWI at admission, a FVHDWI mismatch was present in 56/61 patients with a PWI-DWI mismatch (sensitivity $=92 \%$; 95\% CI, 85\%-99\%). There was no FVH-DWI mismatch in 21/33 patients without PWI-DWI mis-
The high prevalence of FVHs irrespective of their location is likely explained by the fact that patients were imaged within 4.5 hours and had proximal MCA occlusion. Indeed, a lower FVH prevalence has been reported in posterior strokes and in distal occlusions $^{2}$ or when onset-to-MR imaging time increased. ${ }^{1,17}$ Like others, ${ }^{10,20}$ we found fewer FVHs in borderzone areas (M4, M6) than in more proximal areas (insula, M2, M5). This FVH gradient is consistent with an increasing flow rate within leptomeningeal collaterals when moving toward borderzone areas.

Several approaches have been proposed to estimate the extent of FVHs. Some authors distinguished FVHs involving more or less than one-third of the MCA territory or of the hypoperfused area, ${ }^{3,6,21}$ but one has to acknowledge the well-known difficulties in determining this cutoff. Others proposed to count ASPECTS regions with FVH-matching boundaries ${ }^{10,20}$ or grade FVHs according to their sulcal location, ${ }^{22}$ without evaluating the reproducibility of these grading systems. Counting the number of axial FLAIR sections with $\mathrm{FVHs}^{5}$ only provides a rostrocaudal extension of FVHs and is dependent on the section number and thickness. FVH-DWI mismatch offers several advantages over previous estimates of FVH extent. It is simple, reproducible, and feasible at bedside and therefore compatible with timely treatment decisions in acute stroke. The FVH-DWI mismatch focuses on FVHs beyond the boundaries of the cortical DWI lesion, ignoring FVHs adjacent to the DWI lesion. Therefore, only FVHs facing potential tissue at risk of infarct expansion are considered in this definition. The presence of larger amounts of at-risk tissue in the presence of FVH-DWI mismatch is also supported by the results of our univariate analysis, with smaller initial DWI lesions and larger infarct progression after treatment, despite a similar 


\begin{tabular}{|c|c|c|c|}
\hline & $\begin{array}{c}\text { FVH-DWI } \\
\text { Mismatch }(n=102)\end{array}$ & $\begin{array}{c}\text { No FVH-DWI } \\
\text { Mismatch }(n=39)\end{array}$ & $P$ Value \\
\hline \multicolumn{4}{|l|}{ Demographics/risk factors } \\
\hline Age (yr) & $68 \pm 15$ & $68 \pm 14$ & .86 \\
\hline Male & $51(50 \%)$ & $23(59 \%)$ & .34 \\
\hline Hypertension & $53(52 \%)$ & $23(59 \%)$ & .46 \\
\hline Diabetes mellitus & $6(6 \%)$ & $7(18 \%)$ & .03 \\
\hline Hyperlipidemia & $35(34 \%)$ & $15(38 \%)$ & .65 \\
\hline Smoking & $37(36 \%)$ & $11(28 \%)$ & .37 \\
\hline \multicolumn{4}{|l|}{ Characteristics at admission } \\
\hline Systolic BP (mm Hg) & $153 \pm 24$ & $158 \pm 18$ & .31 \\
\hline Diastolic BP $(\mathrm{mm} \mathrm{Hg})$ & $83 \pm 18$ & $83 \pm 17$ & .89 \\
\hline Serum glucose level (mmol/L) & $6.8 \pm 1.6$ & $7.5 \pm 1.9$ & .02 \\
\hline Initial NIHSS score & $16(12-20)$ & $17(15-22)$ & .054 \\
\hline \multicolumn{4}{|l|}{ Initial MRI } \\
\hline Time from onset to initial MRI (min) & $117(87-144)$ & $122(96-159)$ & .16 \\
\hline Available PWI sequence & $68(67 \%)$ & $26(67 \%)$ & 1.00 \\
\hline Terminal ICA occlusion & $39(38 \%)$ & $11(28 \%)$ & .27 \\
\hline Good-to-excellent FLAIR images & $81(79 \%)$ & $24(61 \%)$ & .03 \\
\hline $\mathrm{DWI}_{1}$ volume $(\mathrm{mL})$ & $18(8-35)$ & $108(57-160)$ & $<.001$ \\
\hline PWI-DWI mismatch volume $(\mathrm{mL})^{\mathrm{b}}$ & $70(40-105)$ & $44(33-64)$ & .03 \\
\hline PWI-DWI mismatch ${ }^{b}$ & $56(82 \%)$ & $5(19 \%)$ & $<.001$ \\
\hline Cardioembolic stroke & $61(60 \%)$ & $16(41 \%)$ & .05 \\
\hline \multicolumn{4}{|l|}{ 24-Hour evolution } \\
\hline Follow-up NIHSS score & $11(5-18)$ & $16(10-22)$ & .004 \\
\hline $\mathrm{DWI}_{2}$ volume $(\mathrm{mL})^{\mathrm{d}}$ & $36(19-66)$ & $161(95-230)$ & $<.001$ \\
\hline Relative infarct progression (\%) & $195(112-356)$ & $133(114-165)$ & .001 \\
\hline Complete recanalization ${ }^{\mathrm{c}, \mathrm{d}}$ & $29(29 \%)$ & $12(32 \%)$ & .77 \\
\hline $\mathrm{mRS}$ score at $3 \mathrm{mo}^{\mathrm{e}}$ & $3(1-4)$ & $4(3-6)$ & .001 \\
\hline $\mathrm{mRS}$ score $\leq 2$ at $3 \mathrm{mo}^{\mathrm{e}}$ & $45(47 \%)$ & $9(24 \%)$ & .02 \\
\hline
\end{tabular}

Note:-BP indicates blood pressure.

${ }^{a}$ Numbers (not \%) are mean \pm SD or median (interquartile range)

${ }^{\mathrm{b}}$ Ninety-four patients (PWI-DWI mismatch: $>6$-second Tmax, Volume hypoperfusion $>1.8 \times$ Volume $_{\text {DWII). }}{ }^{19}$

${ }^{c} \mathrm{TICl} 3$.

d 24-hour MRI available in 138 patients.

${ }^{\mathrm{e}} \mathrm{mRS}$ score at 3 months available in 133 patients.

rate of complete recanalization in both groups. These results closely match those from a previous series of 52 patients treated with thrombolysis for MCA occlusion. ${ }^{3}$ Similarly, others concluded that the extent of FVHs was associated with the presence of a PWI-DWI mismatch, though the definition used for the latter was qualitative ${ }^{13}$ or unspecified. ${ }^{8,10}$ Taken together with these previous findings, our results in turn reinforce the view that FVHs beyond the DWI lesion represent markedly impaired hemodynamics in patients with proximal occlusion.

The excellent sensitivity (92\%) of FVH-DWI mismatch for a large PWI-DWI mismatch indicates that the former only rarely missed the latter. This could indicate that the absence of FVHDWI mismatch may obviate PWI to identify patients with large penumbra. However, the moderate specificity (64\%) means that FVH-DWI mismatch as implemented here captures hemodynamic compromises other than just the penumbra. Indeed, if bright vessels on FLAIR effectively represent slow flow in dilated leptomeningeal arterioles in response to cerebrovascular autoregulation, FVHs should overlie not just the penumbral cortex but also the less severely hypoperfused, not at-risk tissue (ie, the "benign" oligemia) and even perhaps surrounding normoperfused but autoregulated tissue. ${ }^{23}$ This hypothesis was strengthened by the post hoc use of a deliberately defined less conservative Tmax threshold ( $>4$ seconds). As expected, specificity increased while sensitivity held up, compared with the standard Tmax of $>6$ seconds. Thus, FVHs beyond the DWI lesion likely detect not just penumbral but also less severely hypoperfused areas. One should, however, keep in mind that the optimum Tmax cut-point separating the penumbra from the oligemia remains somewhat unclear. ${ }^{24}$ However, even though the FVH-DWI mismatch likely overestimates the penumbra, it could serve as a surrogate for PWI-DWI mismatch whenever PWI is unavailable or degraded by artifacts or patients have difficult venous access or contraindications for gadolinium use such as renal failure or contrast hypersensitivity.

Our retrospective study has several limitations. First, our results cannot be generalized to all patients with stroke, especially those with no or distal occlusion. However, we deliberately selected a population of patients with proximal MCA occlusion and visible anterior cerebral artery origin to ensure homogeneity of the potential collateral supply, given the discrepant FVH findings in previous more heterogeneous populations. Second, we were unable to correlate FVHs with collateral flow because patients did not undergo digital subtraction or CT angiography. Third, we defined the PWI-DWI mismatch on the basis of stringent DEFUSE 2 criteria. The accuracy of the FVH-DWI mismatch as a surrogate for PWIDWI mismatch obviously depends on the definition of the latter, which is still debated. Fourth, slight differences in FLAIR parameters among patients in our study are unlikely to have induced major variability in FVH detection. We must, however, be careful in drawing any definite conclusion because differences in the FLAIR sequence, coil system, and magnetic field or among manufacturers may influence FVH visibility.

\section{CONCLUSIONS}

The FVH-DWI mismatch is a novel and promising approach that clearly contains important hemodynamic information, assessable by the naked eye. It could serve as a surrogate to PWI to identify patients with large PWI-DWI mismatches whenever perfusion data are missing. The criteria for the FVH-DWI mismatch model developed here require validation in an independent cohort. If validated, this straightforward MR imaging feature may prove useful for enriching trial cohorts with patients likely to benefit from reperfusion therapies.

Disclosures: Laurence Legrand_RELATED: Grant: Société Française de Radiologie. Marie Tisserand_RELATED: Grant: Fondation pour la Recherche Médicale. Myriam Edjlali-RELATED: Grant: Societe Française de Neurovasculaire. Jean-Louis MasUNRELATED: Board Membership: Bayer, Boehringer-Ingelheim, Bristol-MyersSquibb, Daiichi-Sankyo, Comments: advisory boards; Payment for Lectures (including service on Speakers Bureaus): Bayer, Boehringer-Ingelheim, Bristol-MyersSquibb, Daiichi-Sankyo.

AJNR Am J Neuroradiol 36:269-74 Feb 2015 www.ajnr.org 


\section{REFERENCES}

1. Azizyan A, Sanossian N, Mogensen MA, et al. Fluid-attenuated inversion recovery vascular hyperintensities: an important imaging marker for cerebrovascular disease. AJNR Am J Neuroradiol 2011; 32:1771-75

2. Sanossian N, Saver JL, Alger JR, et al. Angiography reveals that fluidattenuated inversion recovery vascular hyperintensities are due to slow flow, not thrombus. AJNR Am J Neuroradiol 2009;30:564-68

3. Lee KY, Latour LL, Luby M, et al. Distal hyperintense vessels on FLAIR: an MRI marker for collateral circulation in acute stroke? Neurology 2009;72:1134-39

4. Huang X, Liu W, Zhu W, et al. Distal hyperintense vessels on FLAIR: a prognostic indicator of acute ischemic stroke. Eur Neurol 2012; 68:214-20

5. Olindo S, Chausson N, Joux J, et al. Fluid-attenuated inversion recovery vascular hyperintensity: an early predictor of clinical outcome in proximal middle cerebral artery occlusion. Arch Neurol 2012;69:1462-68

6. Pérez de la Ossa N, Hernandez-Perez M, Domenech S, et al. Hyperintensity of distal vessels on FLAIR is associated with slow progression of the infarction in acute ischemic stroke. Cerebrovasc Dis 2012;34:376-84

7. Girot M, Gauvrit JY, Cordonnier C, et al. Prognostic value of hyperintense vessel signals on fluid-attenuated inversion recovery sequences in acute cerebral ischemia. Eur Neurol 2007;57:75-79

8. Ebinger M, Kufner A, Galinovic I, et al. Fluid-attenuated inversion recovery images and stroke outcome after thrombolysis. Stroke 2012;43:539-42

9. Cheng B, Ebinger M, Kufner A, et al. Hyperintense vessels on acute stroke fluid-attenuated inversion recovery imaging: associations with clinical and other MRI findings. Stroke 2012;43:2957-61

10. Hohenhaus M, Schmidt WU, Brunecker P, et al. FLAIR vascular hyperintensities in acute ICA and MCA infarction: a marker for mismatch and stroke severity? Cerebrovasc Dis 2012;34:63-69

11. Schellinger PD, Chalela JA, Kang DW, et al. Diagnostic and prognostic value of early MR imaging vessel signs in hyperacute stroke patients imaged $<3$ hours and treated with recombinant tissue plasminogen activator. AJNR Am J Neuroradiol 2005;26:618-24
12. Kamran S, Bates V, Bakshi R, et al. Significance of hyperintense vessels on FLAIR MRI in acute stroke. Neurology 2000;55:265-69

13. Haussen DC, Koch S, Saraf-Lavi E, et al. FLAIR distal hyperintense vessels as a marker of perfusion-diffusion mismatch in acute stroke. J Neuroimaging 2013;23:397-400

14. Toyoda K, Ida M, Fukuda K. Fluid-attenuated inversion recovery intraarterial signal: an early sign of hyperacute cerebral ischemia. AJNR Am J Neuroradiol 2001;22:1021-29

15. Cosnard G, Duprez T, Grandin C, et al. Fast FLAIR sequence for detecting major vascular abnormalities during the hyperacute phase of stroke: a comparison with MR angiography. Neuroradiology 1999;41:342-46

16. Bang OY, Saver JL, Kim SJ, et al. Collateral flow predicts response to endovascular therapy for acute ischemic stroke. Stroke 2011;42: 693-99

17. Maeda M, Koshimoto Y, Uematsu H, et al. Time course of arterial hyperintensity with fast fluid-attenuated inversion-recovery imaging in acute and subacute middle cerebral arterial infarction. $J$ Magn Reson Imaging 2001;13:987-90

18. Labeyrie MA, Turc G, Hess A, et al. Diffusion lesion reversal after thrombolysis: a MR correlate of early neurological improvement. Stroke 2012;43:2986-91

19. Lansberg MG, Straka M, Kemp S, et al. MRI profile and response to endovascular reperfusion after stroke (DEFUSE 2): a prospective cohort study. Lancet Neurol 2012;11:860-67

20. Kono T, Naka H, Nomura E, et al. The association between hyperintense vessel sign and final ischemic lesion differ in its location. Stroke Cerebrovasc Dis 2014;23:1337-43

21. Gawlitza M, Gragert J, Quaschling U, et al. FLAIR-hyperintense vessel sign, diffusion-perfusion mismatch and infarct growth in acute ischemic stroke without vascular recanalisation therapy. J Neuroradiol 2014;41:227-33

22. Liu W, Xu G, Yue X, et al. Hyperintense vessels on FLAIR: a useful non-invasive method for assessing intracerebral collaterals. Eur $J$ Radiol 2011;80:786-91

23. Muir KW, Buchan A, von Kummer R, et al. Imaging of acute stroke. Lancet Neurol 2006;5:755-68

24. Donnan GA, Baron JC, Ma H, et al. Penumbral selection of patients for trials of acute stroke therapy. Lancet Neurol 2009;8:261-69 